\title{
A Survey of the Practice and Perspectives of Chinese Acupuncturists on Deqi
}

\author{
Yu-lan Ren, ${ }^{1}$ Tai-pin Guo, ${ }^{1}$ Huai-bin Du, ${ }^{1}$ Hua-bin Zheng, ${ }^{2}$ Ting-ting Ma, ${ }^{2}$ \\ Li Fang, ${ }^{3}$ Yu-jie Gao, ${ }^{4}$ Xu-guang Yang, ${ }^{5}$ Xue-zhi Li, ${ }^{6}$ Jing Shi, ${ }^{7}$ Liang Chen, ${ }^{1}$ Yi-wei Liu, ${ }^{1}$ \\ Ru-wen Zhang, ${ }^{1}$ Hui Zheng, ${ }^{1}$ De-hua $\mathrm{Li}^{1}{ }^{1} \mathrm{Xi} \mathrm{Wu},{ }^{1}$ and Fan-rong Liang ${ }^{1}$ \\ ${ }^{1}$ School of Acupuncture Moxibustion and Tuina, Chengdu University of Traditional Chinese Medicine, Chengdu 610075, China \\ ${ }^{2}$ The Affiliated Hospital, Chengdu University of Traditional Chinese Medicine, Chengdu 610075, China \\ ${ }^{3}$ The Third Affiliated Hospital, Zhejiang University of Traditional Chinese Medicine, Hangzhou 310005, China \\ ${ }^{4}$ School of Traditional Chinese Medicine, Ningxia Medical University, Yinchuan 750004, China \\ ${ }^{5}$ School of Acupuncture Moxibustion and Tuina, Henan University of Traditional Chinese Medicine, Zhengzhou 450008, China \\ ${ }^{6}$ School of Traditional Chinese Medicine, Chongqing Medical University, Chongqing 401331, China \\ ${ }^{7}$ Yunnan Province Hospital of Traditional Chinese Medicine, Kunming 650021, China
}

Correspondence should be addressed to Fan-rong Liang; acuresearch@126.com

Received 1 February 2014; Accepted 22 July 2014

Academic Editor: Cun-Zhi Liu

Copyright (C) 2015 Yu-lan Ren et al. This is an open access article distributed under the Creative Commons Attribution License, which permits unrestricted use, distribution, and reproduction in any medium, provided the original work is properly cited.

Deqi refers to the special sensation and reaction sensed mainly by both acupuncturist and patient when a needle was inserted into the acupoints and is considered to be vital to achieve acupuncture effect. For acupuncturist, it is important to judge and control Deqi in clinical practice. However, enough attention is paid to patients' feelings rather than acupuncturists' nowadays. We thus conducted this survey to determine acupuncturists' perspectives about Deqi and to further find the proper way to induce Deqi. A total of 250 questionnaires were sent out to acupuncturists and 202 (80.8\%) were returned. According to the results, most acupuncturists believe that Deqi is vital to obtain preferable clinical effects. The reliability of acupuncturists' Deqi sensation ranks as sinking $>$ tightening $>$ astringent. The reliability of patients' Deqi sensations ranks as sourness $>$ numbness $>$ distention $>$ heaviness $>$ pain. The reliability of influential factors ranks as manipulation $>$ specificity of acupoint $>$ TCM constitution > disease status > patient's psychological condition > acupuncturists' psychological guidance $>$ clinical environment. This study is believed to provide additional evidence to the qualitative and quantitative research of Deqi in the future.

\section{Introduction}

Acupuncture is one of the major treatment modalities in traditional Chinese medicine (TCM). For more than 2500 years of practice, it has been widely accepted by general population in China for its curative effect, wide range of indication, simplicity, and safety in practice. It has gradually become a global therapeutic method in recent decades. According to the theory of traditional acupuncture, the effect of acupuncture is achieved by regulating the channel $Q i$. Therefore, $Q i$ arrival (Deqi), also known as needling sensation, is considered to be closely related to the acupuncture efficacy [1]. In clinical practice, Deqi is measured by the sensation of acupuncturists' finger and patient's reaction. Generally speaking, when Deqi occurs, an acupuncturist may feel sinking (Chen), astringent (Se), and tightness ( Jin) around the needle by his/her fingers. Meanwhile, the patient may sense soreness (Suan), numbness ( $M a)$, distention (Zhang), and heaviness (Zhong) around the acupoint; sometimes, Deqi sensation could be different like coldness, warmness, pain electric-shock feeling, and so forth. However, the intensity and property of acupuncture Deqi may differ as a result of the different physical and psychological conditions of individuals, which made it difficult to be comprehensively applied in clinics. 
Deqi sensation scale, as an important qualitative and quantitative measuring tool for Deqi, was applied to acupuncture clinical trials and mechanism studies recently [2-5]. In 1989, the Vincent Deqi scale was invented with 20 adjectives based on the McGill pain questionnaire. And then there were the Park Deqi scale and the MacPherson Deqi scale followed by $[6,7]$. However, these scales had mainly focused on the patients' sensations but no attention had been paid to the Deqi sensation of acupuncturist. Further, the Southampton Deqi scale was drafted based on the suggestions of both patients and acupuncturists but failed to discriminate the noxious pain sensation from Deqi sensations according to a German trial $[8,9]$. The Massachusetts general hospital acupuncture sensation scale (MASS), which was modified based on the subjective acupuncture sensation scale (SASS) [10], was composed of various needling sensations and had a measurement of the spreading of Deqi and patient's anxiety during needling. It has already been validated as a reliable and valid tool to measure Deqi in healthy young Chinese people [11]. However, till now no standardized, valid, and reliable Deqi scale has been formed due to the lack of sufficient evidence.

In TCM classics, the process to achieve Deqi is also called "Qizhi," which means Qi arrival or Qi obtained through acupuncturist's manipulations after needle insertion. One of the chief indicators of achieving Deqi is the sensation change felt by acupuncturist's fingers. The activation, retention, and spreading of Deqi are closely and directly related to acupuncture manipulation techniques, while recent literature indicates that research attention has been merely paid to the patients' or healthy subjects' needle sensations, with ignorance of the Deqi sensation felt by acupuncturists. Therefore, we conducted this survey by consulting acupuncturists, who are engaging in clinical practice in Chinese hospitals, to explore acupuncturist's perspectives on Deqi and to further understand if there are different views among acupuncturists with different levels of experience.

\section{Methods}

The questionnaire for measuring acupuncture Deqi in acupuncturist was initially designed by 2 senior acupuncture experts, together with 2 clinical acupuncturists and 2 doctoral candidates. All participants of questionnaire design embrace rich experiences in acupuncture treatment with skillful acupuncture manipulation techniques. The current questionnaire we presented in this paper is an autonym questionnaire in Chinese that is finally completed after 5 times revisions according to acupuncture experts suggestions and comments. It contains three parts with 16 items relating to acupuncture Deqi. Part 1 includes 2 single choice questions and 1 open-ended question regarding the acupuncturist's thoughts on the relationship between Deqi and acupuncture efficacy. The questions in part 2 include 2 single choice questions, 3 multiple choice questions, and 2 questions for multiple choices, aimed to identify the proper way to judge Deqi in clinical practice. The questions in part 3 include 4 single choice questions, 2 multiple choice questions, and
1 question for multiple choices, aiming to find the proper manipulation for a better control and guidance of Deqi in clinical practice.

According to the latest literature [12], 90\% acupuncturists thought Deqi was related to clinical efficacy. Based on assumption that it reached $96 \%$ in this study, a sample size of 200 at least is needed to achieve $90 \%$ power to detect a statistical significance by using a two-sided binomial test. The target significance level was 0.05 . Thus, 250 acupuncturists were needed assuming a $25 \%$ dropout rate. A total of 250 questionnaires were sent out to 250 acupuncturists regardless of age or gender in 44 hospitals with express delivery. The included hospitals were geographically distributed in Zhejiang, Jiangxi, Hunan, Shanxi, Qinghai, Sichuan, Yunnan, and Guizhou province and Beijing, Tianjin, and Chongqing municipal city. The rationale for choosing these hospitals in different areas of different directions of China is aiming to avoid deviations caused by the dominance of a specific acupuncture theory or schools in one region. Among them, there were 34 top grade hospitals (77.2\%), and most of them are TCM hospitals, 7 second grade hospitals (16\%), and 3 community hospitals $(6.8 \%)$. All questionnaires were completed by included acupuncturists independently.

The data of questionnaire was collected back-to-back by two researchers. Then, the data was completely and accurately transferred to computerized database for data processing including double data entry, edit checks, data cleaning, coding, and reconciliation. Continuous variables were summarized as means (SDs) and discrete data as frequency and percentage.

\section{Results}

3.1. Participants. 250 questionnaires were sent out, and 202 $(80.8 \%)$ were returned. $49.5 \%$ of the respondents were male. The participants were aged from 19 to 59 years (mean, 33.5), with working years ranging from 1 to 45 years (mean, 9.0 years) (1-5-year experience, $N=96 ; 6-10$-year experience, $N=49 ; 10+$ years of experience, $N=57)$. Regarding the education level, there are 8 with associate degree, 93 participants with bachelor's degree, 73 with master degree, and 18 with Ph.D. degree. Regarding technical title, there were 7 physician assistants, 98 resident doctors, 43 attending physicians, 41 associate physicians, and 13 chief physicians.

\subsection{Perspectives on the Relationship between Deqi and Acu-} puncture Efficacy. As shown in Table 1, regarding the question "whether the Deqi was crucial to clinical efficacy," 194 acupuncturists responded, and $85.57 \%$ of them thought that in most cases Deqi was crucial to clinical efficacy, and $8.76 \%$ thought it was absolutely crucial, while $2.06 \%$ of them stated that in most cases Deqi is not vital to therapy, and $3.61 \%$ of them were not sure. No one chose that Deqi was not related to treatment at all. Regarding the question "whether a higher intensity of Deqi resulted in a more preferable efficacy," most acupuncturists (65.80\%) did not think so, while $34.20 \%$ of the participants agreed. For the reason why the higher Deqi intensity did not yield better efficacy, some 
TABle 1: Perspectives on the relationship between Deqi and acupuncture efficacy.

\begin{tabular}{llc}
\hline Questions & Views & Respondents $(n) /$ all respondents $(n), \%$ \\
\hline & (1) Yes, totally & $17 / 194,8.76 \%$ \\
& (2) Yes, mostly & $166 / 194,85.57 \%$ \\
(1) Do you think Deqi is crucial to acupuncture clinical & (3) No, mostly not & $4 / 194,2.06 \%$ \\
efficacy? & (4) No, totally not & 0 \\
& (5) Uncertainty & $7 / 194,3.61 \%$ \\
\hline (2) Do you think a higher intensity of Deqi results in a & (1) Yes & $66 / 193,34.20 \%$ \\
more preferable efficacy? & (2) No & $127 / 193,65.80 \%$ \\
\hline
\end{tabular}

TABLE 2: The way to judge Deqi.

\begin{tabular}{|c|c|c|}
\hline Questions & Views & $\begin{array}{l}\text { Respondents }(n) / \text { all } \\
\text { respondents }(n), \%\end{array}$ \\
\hline \multirow{3}{*}{$\begin{array}{l}\text { (3) Which way(s) do you prefer to judge the occurrence } \\
\text { of Deqi? (multiple choices) }\end{array}$} & (1) According to sensation of patient's reaction. & $169 / 193,87.56 \%$ \\
\hline & (2) According to sensation of your fingers & $157 / 193,81.35 \%$ \\
\hline & (3) According to facial expression of patient & $66 / 193,36.27 \%$ \\
\hline \multirow{5}{*}{$\begin{array}{l}\text { (4) What is the probability of the case that Deqi has } \\
\text { been felt by your fingers but the patient reported not? }\end{array}$} & (1) Less than $5 \%$ & $50 / 192,26.04 \%$ \\
\hline & (2) $5 \%-10 \%$ & $97 / 192,50.52 \%$ \\
\hline & (3) $10 \%-15 \%$ & $26 / 192,13.54 \%$ \\
\hline & (4) $15-20 \%$ & $13 / 192,6.77 \%$ \\
\hline & (5) More than $20 \%$ & $6 / 192,3.13 \%$ \\
\hline \multirow{2}{*}{$\begin{array}{l}\text { (5) Can you recognize whether the Deqi has appeared } \\
\text { just by your fingers' sensations during manipulations? }\end{array}$} & (1) Yes & $171 / 194,88.14 \%$ \\
\hline & (2) No & $23 / 194,11.86 \%$ \\
\hline \multirow{4}{*}{$\begin{array}{l}\text { (6) What sensations have been felt by your fingers when } \\
\text { Deqi has emerged? (multiple choice) }\end{array}$} & (1) Sinking & $176 / 185,95.14 \%$ \\
\hline & (2) Astringent & $141 / 185,76.22 \%$ \\
\hline & (3) Tightening & $180 / 185,97.30 \%$ \\
\hline & (4) Others & $7 / 185,3.78 \%$ \\
\hline
\end{tabular}

(7) Please rank the acupuncturists' Deqi sensations (those you chose in Question (6) in the order of their reliability for telling the arrival of $Q i$.

/ Shown in Figure 1

\begin{tabular}{llc}
\hline & (1) Sourness & $182 / 196,92.86 \%$ \\
& (2) Numbness & $170 / 196,86.73 \%$ \\
(8) When Deqi occurred, what are the sensations & (3) Distention & $189 / 196,96.43 \%$ \\
reported by patients? (multiple choices) & (4) Heaviness & $139 / 196,70.92 \%$ \\
& (5) Pain & $104 / 196,53.06 \%$ \\
& (6) Others & $7 / 196,3.57 \%$ \\
\hline
\end{tabular}

(9) Please rank the patients' Deqi sensations (those you chose in Question (8)) in the order of their reliability / for telling the arrival of $Q i$.

acupuncturists $(45.67 \%)$ thought it was related to patients' acceptance and tolerability to acupuncture, because a higher intensity possibley resulted in harm of healthy $q i$ or even noxious stimulation. $26.77 \%$ thought it was attributed to individual difference. 3.94\% thought it was complicated. And $23.62 \%$ did not give any answers.

3.3. The Way to Judge Deqi. As shown in Table 2, Question (3) is a multiple choice question, and acupuncturist could make one or more choices. $87.56 \%$ of the acupuncturists said they jndged the occurrence of Deqi according to patient's sensation, $81.35 \%$ based on their personal sensation felt by fingers, and $36.27 \%$ according to the facial expression of patient. Question (4) demonstrated that there is only a small chance when Deqi was felt by acupuncturist but not by patient. The results showed that $50.52 \%$ of acupuncturists thought the probability was $5 \%-10 \%$, and $26.04 \%$ thought it is less than 5\%. Regarding Question (5), 88.14\% of the acupuncturists could tell whether the Deqi was achieved just by their manipulating fingers' sensation regardless of patient's sensation.

Question (6) was also a multiple choice question. The highest frequency of any sensation felt by the acupuncturists during Deqi was tightening (97.30\%), followed by 


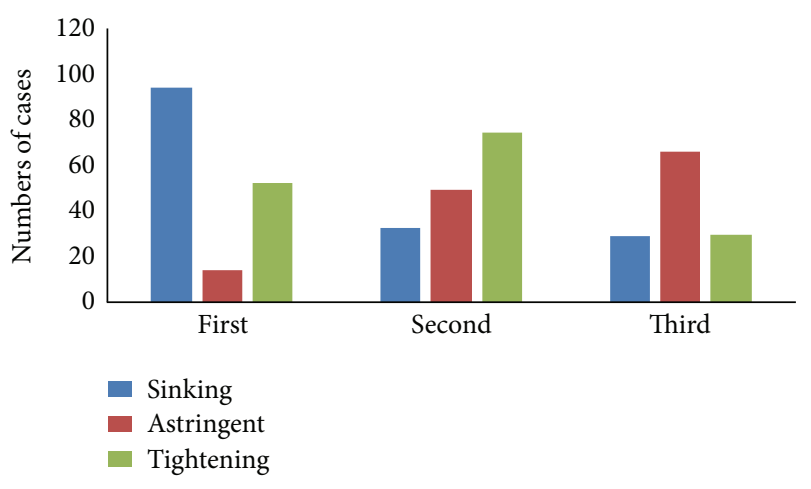

FIgURE 1: The reliability order of acupuncturists' Deqi sensations in Question (6).

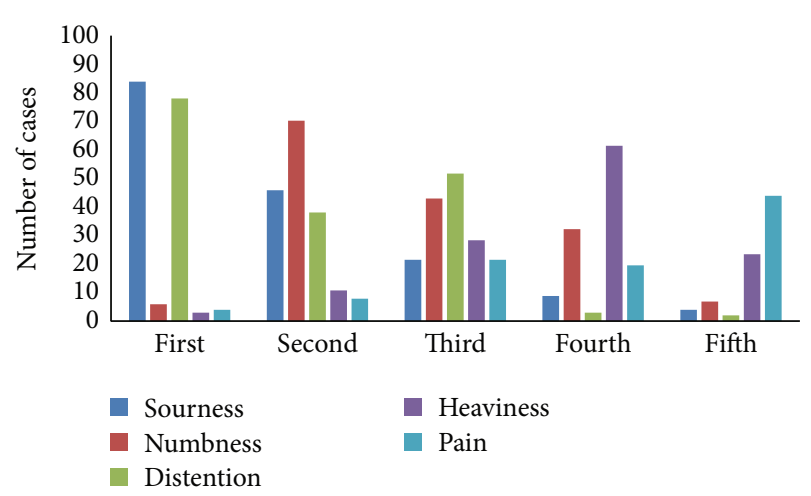

FIGURE 2: The reliability order of patients' Deqi sensations in Question (8).

sinking (95.14\%), astringent (76.22\%), and others (3.78\%). In Question (7), acupuncturists ranked the reliability of telling Deqi according to their sensations shown in Question (6) in sequence. Figure 1 demonstrated that acupuncturist believed that sinking was ranked at the first place, while tightening the second, and astringent the third, in the reliability of telling Deqi based on their personal sensations.

Answers to Question (8) demonstrated that main sensations of Deqi reported by patients were distention (96.43\%), soreness $(92.86 \%)$, numbness $(86.73 \%)$, heaviness (70.92\%), and pain $(53.06 \%)$. In order to determine the reliability of telling Deqi by patient's subjective sensation, acupuncturists were required to answer Question (9). The result in Figure 2 showed that acupuncturist believed that soreness was ranked at the first place, while numbness the second, and distention the third, followed by heaviness and pain, in the reliability of telling Deqi based on patients' subjective sensations.

3.4. The Way to Control Deqi. As shown in Table 3, Question (10), regarding the influential factors of Deqi during clinical practices, was a multiple choice question. The main influential factors of Deqi were manipulation (98.43\%), patient's body constitution (95.29\%), acupoint (87.43\%), the state of illness $(80.10 \%)$, and psychological condition of patient (68.06\%). The importance and reliability of Deqi's influential factors to Deqi was ranked in the order as manipulation

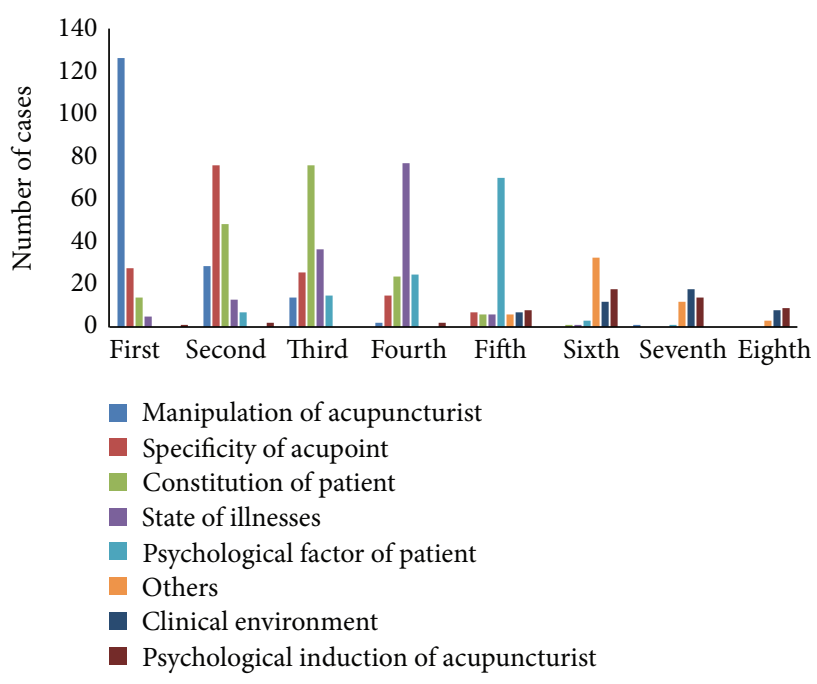

FIGURE 3: The order of influencing factors of Deqi in Question (16).

of acupuncturist $>$ specificity of acupoints $>$ constitution of patient $>$ status of illness $>$ pathogenetic condition of patient, as shown in Figure 3.

Regarding Question (12), the most frequently used manipulation to promote Deqi sensations was the combination of rotating, lifting, and thrusting (72.54\%), and supplementary manipulations such as scrape, shake, and press were also used by some acupuncturists (17.62\%), while just one kind of manipulation was seldom applied. The questions (13) showed that most acupuncturists (74.21\%) believed that the depth of insertion to elicit Deqi sensation should be based on the patient's conditions, and $25.79 \%$ thought that deep insertion was easier, but few chose shallow insertion. In response to the question of whether the strong sensation produced by rotating the needle in single direction was true Deqi or not, $46.87 \%$ acupuncturists agreed with this point. According to Question (15), the majorities of acupuncturists (98.45\%) are able to induce Deqi right at the moment of needle insertion, even without needle manipulation. But the possibility was not high for $21.05 \%$ of acupuncturists thought it $<20 \%$ and $38.42 \%$ of acupuncturists thought $20 \%-40 \%$, indicated in Question (16).

\section{Discussions}

4.1. Deqi Is Crucial to Acupuncture Efficacy. In our study, 94.33\% acupuncturists stated Deqi was crucial to clinical efficacy. Of these, $85.57 \%$ thought it was crucial in most cases while $8.76 \%$ thought it was always crucial. No one denied its importance completely. A recently randomized controlled trial (RCT) of Bell's palsy demonstrated that the strong Deqi could result in better outcomes [2], while another study stated no pain relief in osteoarthritis patients [13]. Regarding the intensity, the majority of acupuncturists did not believe that a stronger intensity would increase the benefits while $34.20 \%$ agreed. This result was similar to the results reported by Han [14], who showed that a low frequency $(2 \mathrm{~Hz})$ had better effects on easing pain than a high frequency $(100 \mathrm{~Hz})$. There 
Table 3: The way to control Deqi.

\begin{tabular}{|c|c|c|}
\hline Questions & Views & $\begin{array}{l}\text { Respondents }(n) / \text { all } \\
\text { respondents }(n), \%\end{array}$ \\
\hline \multirow{7}{*}{$\begin{array}{l}\text { (10) What is the influential factors of Deqi during your } \\
\text { clinical practices? (multiple choices) }\end{array}$} & (1) Manipulation of acupuncturist & $188 / 191,98.43 \%$ \\
\hline & (2) Specificity of acupoint & $167 / 191,87.43 \%$ \\
\hline & (3) Constitution of patient & $182 / 191,95.29 \%$ \\
\hline & (4) State of illnesses & $153 / 191,80.10 \%$ \\
\hline & (5) Psychological factor of patient & $130 / 191,68.06 \%$ \\
\hline & (6) Clinical environment & $53 / 191,27.75 \%$ \\
\hline & $\begin{array}{l}\text { (7) Psychological induction of } \\
\text { acupuncturist }\end{array}$ & $54 / 191,28.27 \%$ \\
\hline $\begin{array}{l}\text { (11) Please rank Deqi's influential factors (those you } \\
\text { chose in Question (10)) in order according to their } \\
\text { reliability and importance. }\end{array}$ & 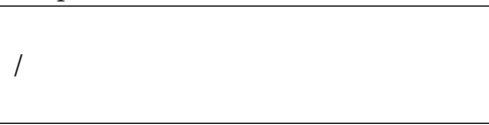 & Showed in Figure 3 \\
\hline \multirow{6}{*}{$\begin{array}{l}\text { (12) Which manipulation is the most commonly used to } \\
\text { promote the arrival of Deqi sensations? }\end{array}$} & (1) Lifting and thrusting & $12 / 193,6.22 \%$ \\
\hline & (2) Rotating & $2 / 193,1.04 \%$ \\
\hline & $\begin{array}{l}\text { (3) Combination of rotating, lifting, } \\
\text { and thrusting }\end{array}$ & $140 / 193,72.54 \%$ \\
\hline & $\begin{array}{l}\text { (4) Supplementary manipulations like } \\
\text { scrape, shake, and so forth. }\end{array}$ & $4 / 193,1.04 \%$ \\
\hline & (5) Combination of (3) and (4) & $34 / 193,17.62 \%$ \\
\hline & (6) Others & $1 / 193,0.52 \%$ \\
\hline \multirow{3}{*}{$\begin{array}{l}\text { (13) For deep or shallow insertion, which one more } \\
\text { easily elicits Deqi sensation according to your } \\
\text { experiences? }\end{array}$} & (1) Deep & $49 / 190,25.79 \%$ \\
\hline & (2) Shallow & 0/190, $0 \%$ \\
\hline & (3) Depending on patients' conditions & $141 / 190,74.21 \%$ \\
\hline \multirow{2}{*}{$\begin{array}{l}\text { (14) For the strong sensations caused by rotating with } \\
\text { one single direction, does this feeling belong to Deqi? }\end{array}$} & (1) Yes & $90 / 192,46.87 \%$ \\
\hline & (2) No & $102 / 192,53.13 \%$ \\
\hline \multirow{2}{*}{$\begin{array}{l}\text { (15) Have Deqi ever occurred as soon as the needle was } \\
\text { inserted without any manipulations? }\end{array}$} & (1) Yes & $190 / 193,98.45 \%$ \\
\hline & (2) No & $3 / 193,1.55 \%$ \\
\hline \multirow{5}{*}{$\begin{array}{l}\text { (16) If (15) has occurred, what is the approximate } \\
\text { occurrence rate? }\end{array}$} & (1) Less than $20 \%$ & $40 / 190,21.05 \%$ \\
\hline & (2) $20 \%-40 \%$ & $73 / 190,38.42 \%$ \\
\hline & (3) $40 \%-60 \%$ & $55 / 190,28.95 \%$ \\
\hline & (4) $40-80 \%$ & $19 / 190,10 \%$ \\
\hline & (5) More than $80 \%$ & $3 / 190,1.58 \%$ \\
\hline
\end{tabular}

is no doubt that Deqi is crucial to clinical efficacy in our study. However, future clinical trials are required to confirm this.

\subsection{Deqi and Acupuncture Manipulations. Our investigation} showed that the most significant influencing factors of Deqi were the acupuncturist's manipulation. Acupuncturists' manipulation was also reported to be the most important influencing factor in clinical studies. It is accepted that manipulation could induce the release of Deqi and promote the degree of Deqi or alleviate the strong Deqi sensation. It is also known that different types of manipulation can result in different Deqi sensations. For promoting or controlling Deqi, acupuncturists preferred to use the combined manipulations of rotating, lifting, and thrusting and also used the supplemental manipulations such as scraping and shaking. As the issue of whether the strong feeling caused by rotating with one single direction was due to Deqi, acupuncturists themselves did not come to an agreement. Sometimes, nearly all acupuncturists have experienced that Deqi occurred just as the needle was inserted without any other manipulations, but the probability was very low.

Different depth of insertion has aroused considerable debate, and more researchers favor the idea that with deep insertion it is easier to produce Deqi sensations. Functional magnetic resonance imaging (fMRI) has shown that deep electroacupuncture on GB34 and GB35 could generate stronger Deqi sensations and more effectively modulate the pain-related neuromatrix than shallow electroacupuncture [15]. Other studies also proved that deep acupuncture could result in higher Deqi sensations scores [16] and increase the skin and muscle blood flow [17] in healthy subjects. However, some studies report the opposite views. A fMRI research declared no significant differences in the blood oxygen level dependent (BOLD) responses by the deep and shallow stimulations [18]. RCT also showed that the same effects were achieved by both deep and superficial acupuncture in 
idiopathic anterior knee pain patients [19]. Our questionnaire results demonstrated that no one thought the shallow insertion made it easier to produce Deqi sensations, and $25.79 \%$ of the acupuncturists stated that deep insertion made it easier, while the majority of the acupuncturists (74.21\%) explained that it depended on the patients' conditions and was not relevant to the depth of insertion. The shallow stimulation usually means that the needle tip reaches the subcutaneous tissue, while the deep stimulation may arrive at the muscular or nervous tissues. Deqi sensation may not be completely relevant to the deep tissues. One study provided evidence that Deqi was not relevant to the deep median nerve contact nor median nerve penetration during needling in P6 point with ultrasound measurement [20]. Another study also explained that it depended on the patients' conditions and no relevance was attributed to the depth of insertion because in healthy subjects the skin and muscle blood flow increased with no significance comparing deep and shallow stimulation, but in fibromyalgia patients there were significant differences [5].

4.3. The Deqi Sensation of Acupuncturists and Patients. It seems that the feelings of patient and acupuncturist are often used to judge whether Deqi has been generated or not, but few researches have demonstrated the real details of judgment. The results of this study showed that the majority of acupuncturists recognized whether the Deqi had been achieved just by their fingers' sensations during manipulations. These sensations were mostly tightening, sinking, and astringent, which were similar as those described in textbooks [1]. Interestingly, the Deqi sensations experienced by fingers were ranked as sinking, tightening, and astringent according to the reliability. In our survey, the patients' sensations were mainly described as soreness (Suan), numbness ( $M a)$, distention (Zhang), heaviness (Zhong), and pain (Tong). The results showed the order of frequency as distention, soreness, numbness, heaviness, and pain, and the orders of its reliability level scores ranked as soreness $>$ numbness $>$ distention $>$ heaviness $>$ pain. For the top three sensations, although there were different words and expressions to describe Zhang, Suan, and Ma, some previous researchers also demonstrated similar results [21-24]. So, Deqi sensation in both acupuncturist and patient, occurrence probability, and the order of reliability were revealed. These results may provide some advice for further quantification studies for Deqi.

4.4. Influencing Factors of Deqi. Except for acupuncturist's manipulation, the common influencing factors of Deqi included the specificity of acupoint, the constitution of patient, and the patients'psychological factor as shown in our study. For the specificity of the acupoint, studies had shown that it existed and was related to clinical effects closely [25], but it needs further clinical study to determine its direct relationship with Deqi. Yet for all that, according to common sense, the feeling of pain is evident with needling the terminal points on the four limbs, while the soreness and distention are noted in thick muscle points. Meanwhile, the conditions of patient such as constitution, illness state, and psychology were considered as the important factors to Deqi in Chinese ancient and modern literatures [26-28]. As noted in "Ling Shu Jing," the speed of Deqi emergence is faster in patient with yang excess constitution than the one with yin excess. Compared with healthy volunteers, patients suffering chronic pain tended to acquire a much stronger Deqi sensation [29]. Some researchers believe that Deqi is the brain awareness and consciousness because the sensations of the subjects were the same between sham laser acupuncture and true laser [30]. However, in the classical literature such as the "Huangdi Neijing" and the "Zhenjiu Dacheng," psychological factors are important with Deqi, not only in the process of acupuncture but also in influencing the clinical outcomes. Clinical trials revealed that Bell's palsy patients with the personality factors of excitability, sociability, braveness, and intellectuality had an easier time to gain Deqi [31], and the anxiety and dominance were correlated with the treatment effects of primary dysmenorrhea [32]. In addition, the anxiety also affected the heart rate variability in healthy subjects [33].

4.5. Limitations. The main limitation of this survey is that the contents are designed according to Chinese acupuncturist's customs, including manipulations, acupuncturist's needling sensations, patient's sensations, and the factors of Deqi, and some items may be difficult to understand and do not conform with international conventions. Most of the acupuncturists came from the grade-three general province hospitals, and opinions from lower grade hospitals were insufficient. The results are from acupuncturists' general perspectives; a supplementary survey of the patients' views is necessary.

\section{Conclusions}

Our survey demonstrates that Deqi is important to clinical effects according to the acupuncturist's views. The integrated manipulations are the most common way to promote Deqi sensation. The reliability of primary acupuncturist fingers' Deqi sensations ranks as sinking > tightening >astringent. The reliability of primary patients' Deqi sensations ranks as sourness $>$ numbness $>$ distention $>$ heaviness $>$ pain as reported by patients. The reliability of primary patient's Deqi sensation factors ranks as manipulation>specificity of acupoint $>$ TCM constitution $>$ disease status $>$ patient's psychology $>$ acupuncturist's psychological hint $>$ clinical environment.

In short, this paper shows the perspectives of Chinese acupuncturists on Deqi. The results may provide some evidences to the qualitative and quantitative research of Deqi. To formulate and evaluate a Deqi sensation scale, it may be better to include both of the sensations of acupuncturist and patient on the basis of the credible rank. In clinical research and practice, the influential factors of Deqi should be considered.

\section{Conflict of Interests}

The authors declare that there is no conflict of interests in this paper. 


\section{Authors' Contribution}

Yu-lan Ren and Tai-pin Guo contributed equally to this paper.

\section{Acknowledgments}

This survey was funded by a Grant from the State Key Development Program for Basic Research of China (973 Program, no. 2012CB518501) and the Transformation Platform Construction Program for Science and Technology Achievement of the Sichuan Province (no. 2012FZ0082). The authors thank Zhan-xin Chen (Qinghai Province Hospital of TCM), Hai-yuan Yan (Shaanxi Yulin Hospital of TCM), Xu Du (Shanxi University of TCM), Tian-zhong Peng (Jiangxi Hongdu Hospital of TCM), Meng Kong (Yunnan Yanshan Hospital of TCM), Yue Fan (Yunnan Zhaotong Hospital of TCM), Wen Yao (Hunan Province Hospital of TCM), Haichun Ji (Zhejiang Taizhou Hospital of TCM), Jian-feng Li (The Fourth People's Hospital of Jiangyou), and Qing-wei Shi (Chengdu Wenjiang Hospital of TCM) for assisting in the distribution and collection of the questionnaires.

\section{References}

[1] F. R. Liang and J. P. Zhao, Acupuncture and Moxibustion, People's Medical Publishing House, Beijing, China, 2012.

[2] S. Xu, B. Huang, C. Zhang et al., "Effectiveness of strengthened stimulation during acupuncture for the treatment of Bell palsy: a randomized controlled trial," CMAJ, vol. 185 , no. 6 , pp. $473-$ 479, 2013.

[3] J. E. Park, Y. H. Ryu, Y. Liu et al., "A literature review of de qi in clinical studies," Acupuncture in Medicine, vol. 31, no. 2, pp. 132-142, 2013.

[4] J. Chen, G. Li, G. Zhang, Y. Huang, S. Wang, and N. Lu, "Brain areas involved in acupuncture needling sensation of de qi: a single-photon emission computed tomography (SPECT) study," Acupuncture in Medicine, vol. 30, no. 4, pp. 316-323, 2012.

[5] M. Sandberg, L. Lindberg, and B. Gerdle, "Peripheral effects of needle stimulation (acupuncture) on skin and muscle blood flow in fibromyalgia," European Journal of Pain, vol. 8, no. 2, pp. 163-171, 2004.

[6] J. Park, H. Lee, S. Lim et al., "Deqi sensation between the acupuncture-experienced and the Naïve: a Korean study II," American Journal of Chinese Medicine, vol. 33, no. 2, pp. 329337, 2005.

[7] H. MacPherson and A. Asghar, "Acupuncture needle sensations associated with De Qi: a classification based on experts' ratings," Journal of Alternative and Complementary Medicine, vol. 12, no. 7, pp. 633-637, 2006.

[8] P. White, F. Bishop, H. Hardy et al., "Southampton needle sensation questionnaire: development and validation of a measure to gauge acupuncture needle sensation," Journal of Alternative and Complementary Medicine, vol. 14, no. 4, pp. 373-379, 2008.

[9] D. Pach, C. Hohmann, R. Lüdtke, F. Zimmermann-Viehoff, C. M. Witt, and C. Thiele, "German translation of the Southampton Needle Sensation questionnaire: use in an experimental acupuncture study," Forschende Komplementarmedizin, vol. 18, no. 6, pp. 321-326, 2011.

[10] J. Kong, D. T. Fufa, A. J. Gerber et al., "Psychophysical outcomes from a randomized pilot study of manual, electro, and sham acupuncture treatment on experimentally induced thermal pain," Journal of Pain, vol. 6, no. 1, pp. 55-64, 2005.

[11] D. T. W. Yu, A. Y. M. Jones, and M. Y. C. Pang, "Development and validation of the chinese version of the massachusetts general hospital acupuncture sensation scale: an exploratory and methodological study," Acupuncture in Medicine, vol. 30, no. 3, pp. 214-221, 2012.

[12] H. W. Yuan, L. X. Ma, P. Zhang et al., "An exploratory survey of deqi sensation from the views and experiences of Chinese patients and acupuncturists," Evidence-Based Complementary and Alternative Medicine, vol. 2013, Article ID 430851, 8 pages, 2013.

[13] P. White, P. Prescott, and G. Lewith, "Does needling sensation (de qi) affect treatment outcome in pain? Analysis of data from a larger single-blind, randomised controlled trial," Acupuncture in Medicine, vol. 28, no. 3, pp. 120-125, 2010.

[14] J. Han, "Acupuncture: neuropeptide release produced by electrical stimulation of different frequencies," Trends in Neurosciences, vol. 26, no. 1, pp. 17-22, 2003.

[15] J. Zhang, X. Cao, J. Li, W. Tang, H. Liu, and X. Feng, "Neuronal specificity of needling acupoints at same meridian: a control functional magnetic resonance imaging study with electroacupuncture," Acupuncture and Electro-Therapeutics Research, vol. 32, no. 3-4, pp. 179-193, 2007.

[16] A. Benham, G. Phillips, and M. I. Johnson, "An experimental study on the self-report of acupuncture needle sensation during deep needling with bi-directional rotation," Acupuncture in Medicine, vol. 28, no. 1, pp. 16-20, 2010.

[17] M. Sandberg, T. Lundeberg, L. G. Lindberg, and B. Gerdle, "Effects of acupuncture on skin and muscle blood flow in healthy subjects," European Journal of Applied Physiology, vol. 90, no. 1-2, pp. 114-119, 2003.

[18] H. MacPherson, G. Green, A. Nevado et al., "Brain imaging of acupuncture: comparing superficial with deep needling," $\mathrm{Neu}$ roscience Letters, vol. 434, no. 1, pp. 144-149, 2008.

[19] J. Näslund, U. Näslund, S. Odenbring, and T. Lundeberg, "Sensory stimulation (acupuncture) for the treatment of idiopathic anterior knee pain," Journal of Rehabilitation Medicine, vol. 34, no. 5, pp. 231-238, 2002.

[20] K. Streitberger, U. Eichenberger, A. Schneider, S. Witte, and M. Greher, "Ultrasound measurements of the distance between acupuncture needle tip at P6 and the median nerve," Journal of Alternative and Complementary Medicine, vol. 13, no. 5, pp. 585591, 2007.

[21] K. K. S. Hui, E. E. Nixon, M. G. Vangel et al., "Characterization of the "deqi" response in acupuncture," BMC Complementary and Alternative Medicine, vol. 7, article 33, 2007.

[22] J. J. Mao, J. T. Farrar, K. Armstrong, A. Donahue, J. Ngo, and M. A. Bowman, "De qi: Chinese acupuncture patients' experiences and beliefs regarding acupuncture needling sensationan exploratory survey," Acupuncture in Medicine, vol. 25, no. 4, pp. 158-165, 2007.

[23] K. Zhou, J. Fang, X. Wang et al., "Characterization of De Qi with electroacupuncture at acupoints with different properties," Journal of Alternative and Complementary Medicine, vol. 17, no. 11, pp. 1007-1013, 2011.

[24] S.-U. Park, C.-N. Ko, H.-S. Bae et al., "Short-term reactions to acupuncture treatment and adverse events following acupuncture: a cross-sectional survey of patient reports in Korea," The Journal of Alternative and Complementary Medicine, vol. 15, no. 12 , pp. 1275-1283, 2009. 
[25] E. M. Choi, F. Jiang, and J. C. Longhurst, "Point specificity in acupuncture," Chinese Medicine, vol. 7, article 4, 2012.

[26] Z. J. Li, F. Zeng, J. Yang et al., "Comments on influence of different functional status of the body on clinical effects of acupuncture therapy," Zhen Ci Yan Jiu, vol. 38, no. 5, pp. 428431, 2012.

[27] J. Li, Y. Q. Liu, C. H. Li et al., "Discussion on the influence of factors related organic on Deqi in acupuncture treatment," Zhongguo Zhen Jiu, vol. 33, no. 4, pp. 378-380, 2013.

[28] F. C. Lin and F. Fei, "Influence of five-state persons in Huangdineijing on obtaining qi," Journal of Beijing University of Traditional Chinese Medicine, vol. 36, no. 2, pp. 90-91, 2013.

[29] P. White, G. Lewith, and P. Prescott, "Should we recruit patients or healthy volunteers for acupuncture studies of chronic pain?" Clinical Journal of Pain, vol. 23, no. 8, pp. 714-719, 2007.

[30] N. Salih, P. I. Bäumler, M. Simang, and D. Irnich, "Deqi sensations without cutaneous sensory input: results of an RCT," BMC Complementary and Alternative Medicine, vol. 10, article 81, 2010.

[31] J. Mei, S. Gao, and G. Chen, "Relationship between neuropsychological factors and effect of acupuncture in treating Bell's palsy," Zhongguo Zhong Xi Yi Jie He Za Zhi, vol. 30, no. 10, pp. 1026-1029, 2010.

[32] J. Xiong, F. Liu, W. Wang, and G. Huang, "[Efficacy impacts of psychological factors on primary dysmenorrhea treated with acupuncture]., Zhongguo Zhen Jiu, vol. 31, no. 6, pp. 493-497, 2011.

[33] V. Vickland, C. Rogers, A. Craig, and Y. Tran, "Anxiety as a factor influencing physiological effects of acupuncture," Complementary Therapies in Clinical Practice, vol. 15, no. 3, pp. 124$128,2009$. 


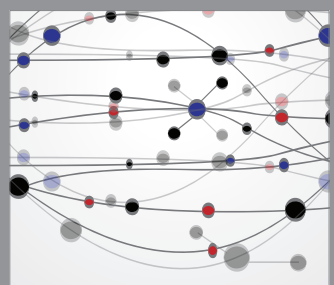

The Scientific World Journal
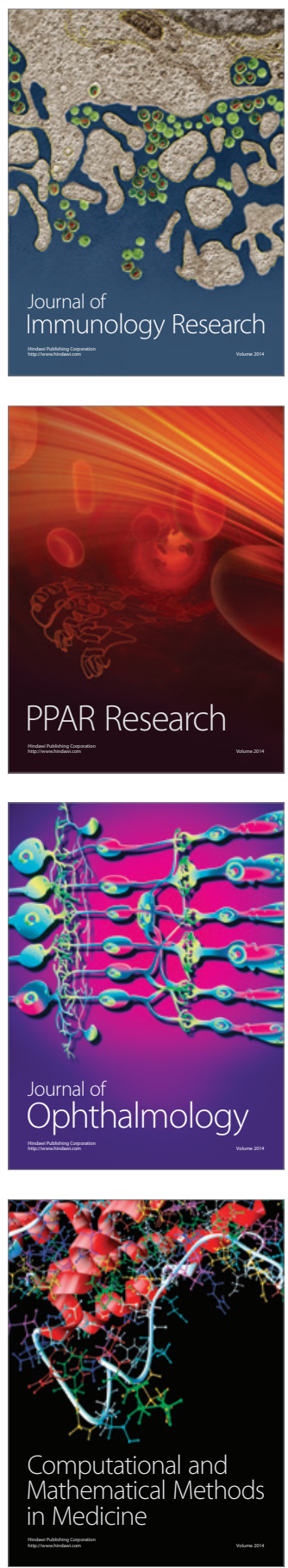

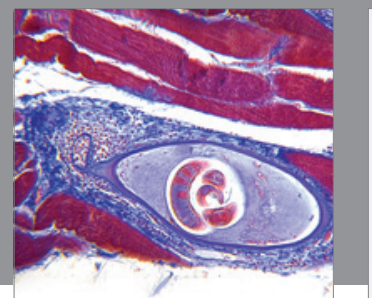

Gastroenterology

Research and Practice
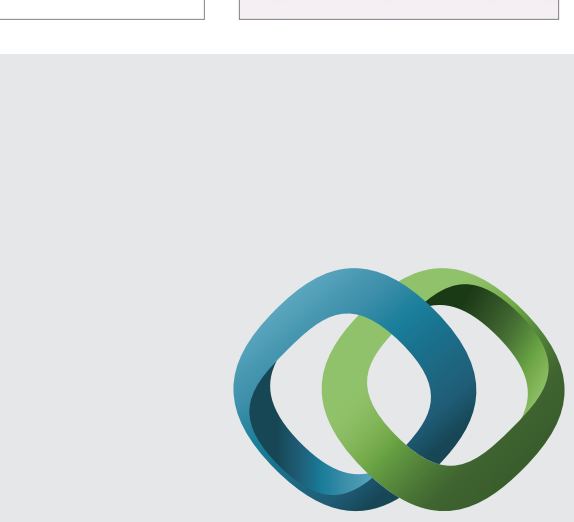

\section{Hindawi}

Submit your manuscripts at

http://www.hindawi.com
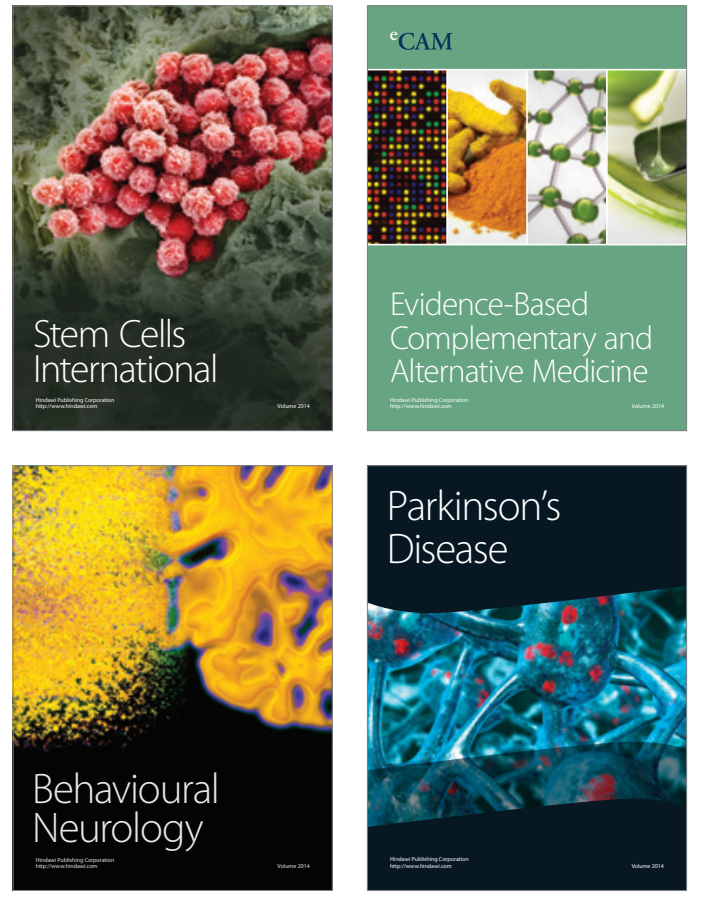
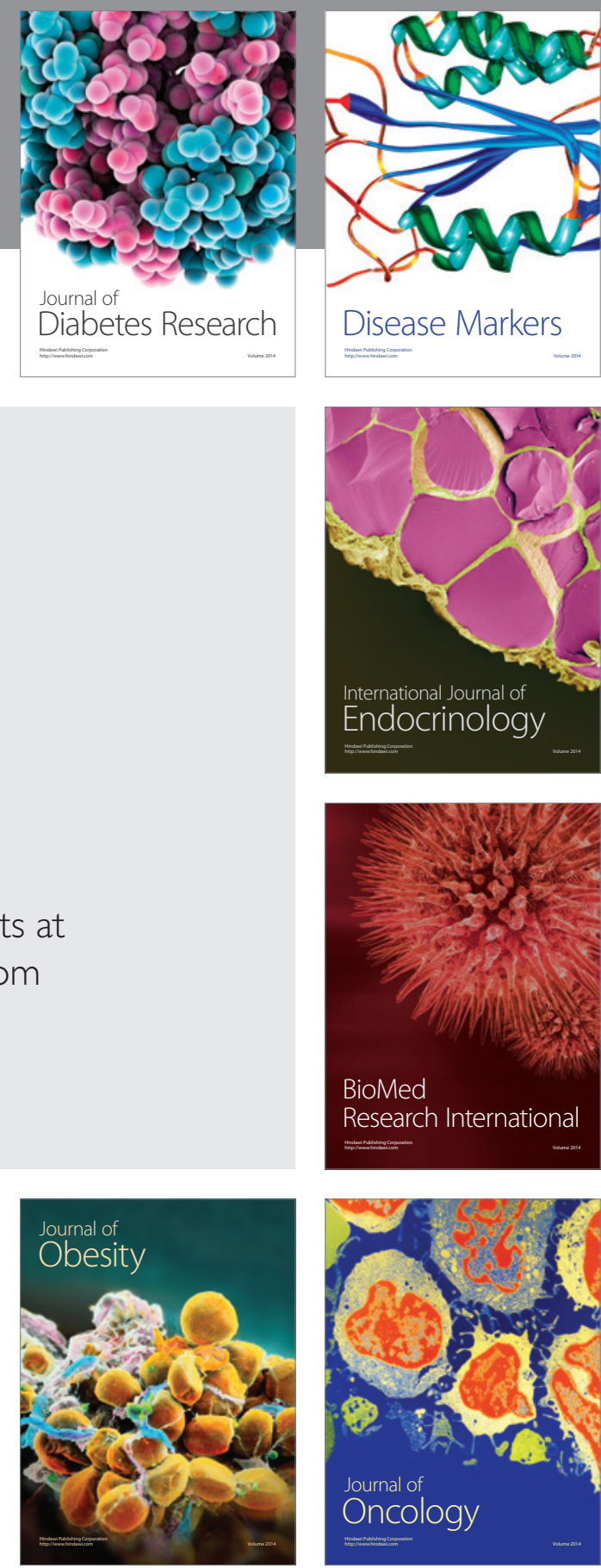

Disease Markers
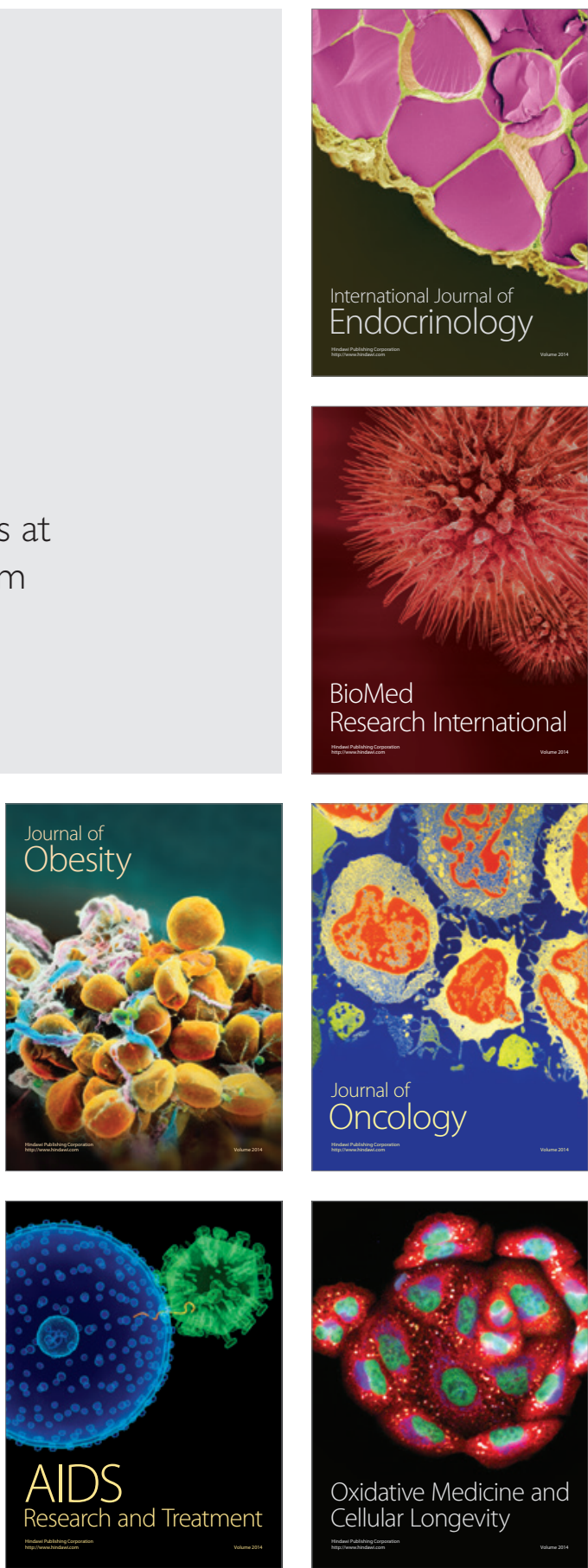